\title{
Experimental and Theoretical Study of Light Propagation in Suspended-Core Optical Fiber
}

\author{
M. Grabka, B. Wajnchold, S. Pustelny, W. Gawlik \\ Center for Magneto-Optical Research, Institute of Physics, Jagiellonian University \\ Reymonta 4, PL-30-059 Kraków, Poland \\ K. Skorupski And P. Mergo \\ Department of Optical Fibres Technology, Faculty of Chemistry, Marie Curie-Skłodowska University \\ pl. M. Curie-Skłodowskiej 3, PL-20-031 Lublin, Poland \\ Dedicated to memory of Jan Wójcik
}

\begin{abstract}
We analyze coupling and propagation of light through a suspended-core microstructured optical fiber. It is experimentally demonstrated that light-coupling efficiency and mode distribution strongly depend on relative position of the fiber's core and a light beam and light polarization. The experimental results are supported with numerical simulations. The developed numerical model confirmed all the observed dependences.
\end{abstract}

PACS numbers: 42.70.Qs, 42.81.Qb, 42.81.-i, 42.81.Pa, 07.60.Vg

\section{Introduction}

Microstructured optical fibers (MOFs) are two-dimensional photonic crystals that have a modulation of their refractive index introduced via arrays of air-holes arranged in periodic structures extending along the fibers' lengths [1]. Their invention in the mid-1990s revolutionized the field of fiber optics by allowing a possibility of tailoring the fibers' properties, in particular their dispersion and modal area. These properties may be engineered by a careful choice of a fiber structure: arrangement of the holes, their pitch and diameters [2]. Control over the fibers' optical properties makes them particularly attractive for such applications as fiber-optic communications, fiber lasers [3], nonlinear devices [4], etc. Moreover, strong confinement of the light in fiber's air-holes enables ultrastrong coupling between light and a medium filling the holes. This allows one to use these fibers as ultrasensitive gas or liquid sensors while strongly reducing the sample volume required for the measurement [5].

Based on the geometrical structure, the MOFs can be divided into two groups: hollow-core (HC) and solid-core (SC) fibers. In the HC MOFs, the guiding mechanism employs the generation of a photonic bandgap in the fiber's cladding that arises due to modulation of the cladding's refractive index. In that case, even with a refractive index of the cladding higher than that of the core, light is trapped and guided through the HC. This allows confinement of up to $99 \%$ of transmitted power in the core, which is very attractive for optical spectroscopy. A significant drawback of the HC MOFs, however, is a limited spectral range, typically narrower than $100 \mathrm{~nm}$, for which the photonic bandgap is generated. Thus, the HC MOFs are very well suited for a narrow-band chemical sensing. On the contrary, a broad-band sensing is possible with the SC MOFs [6]. In such fibers, light is guided through the SC based on total internal reflection (index-guiding) and transmission is defined by the loss of the fiber material. In these fibers light penetrates holes as an evanescent field, hence only a small fraction of the total power (typically no more than $10 \%$ ) is transmitted through the holes.

A particular example of the SC MOFs is a suspended-core fiber (SCF) [7]. The fiber consists of a silica rod that has a diameter comparable with the light wavelength $(\approx 1 \mu \mathrm{m})$ suspended with three thin (on the order of a hundred $\mathrm{nm}$ ) struts attached to a solid cladding of the fiber. Since the struts are much longer than the core diameter, the core is surrounded by large air-holes. Due to the large refractive index contrast between the solid core and the air-holes, light is confined in the core and typically a few percent of its total power penetrates the holes as an evanescent wave $[8,9]$. Independently of the strong confinement of the light, application of the fibers as chemical sensors was demonstrated $[10,11]$.

In this article we theoretically and experimentally analyze the propagation of light in the SCF. The goal is to understand the coupling of light in the fiber's core and light propagation through the fiber. The paper covers three general topics: fiber's mode structure, numerical aperture NA, and mode-coupling efficiency, which be used in our future research.

\section{Experimental setup and numerical simulations}

The layout of our experimental setup is shown in Fig. 1. A frequency stabilized light of $795 \mathrm{~nm}$ is emitted from an extended cavity diode laser. The light is generated in a single longitudinal mode (spectral width below $1 \mathrm{MHz}$ ) and many transverse modes. In order to achieve a beam in a single transverse mode, spatial filtering is performed. 
It is realized by sending the light beam through a single-mode polarization preserving fiber (Panda type). As a result, a well-shaped Gaussian beam of $2 \mathrm{~mW}$ light power is obtained.

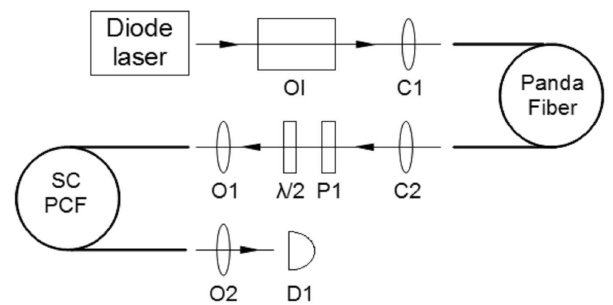

Fig. 1. Experimental setup. OI - optical insulator, C1, C2 - couplers, P1 - polarizer, $\lambda / 2$ - half-wave plate, $\mathrm{O} 1, \mathrm{O} 2$ - objectives $60 \times, \mathrm{SC} \mathrm{PCF}$ - examined fiber, D1 - power meter. In case of numerical aperture measurements, objective $\mathrm{O} 2$ is replaced with D1 mounted on the goniometer table.

The beam is coupled to an SCF fabricated with stacked-and-draw method [12]. A fiber's core diameter is equal to $1.35 \mu \mathrm{m}$ and struts width is about $130 \mathrm{~nm}$ (Fig. 2). Coupling of the light into such a small core is possible due to installation of the fiber in a holder attached to a precise $x-y-z$ transition table (NanoMax300 from ThorLabs). It enables positioning of the fiber's end with sub-micrometer precision and its accurate placement in the focus of the coupling objective of a $60 \times$ magnification and high numerical aperture $(\mathrm{NA}=0.85)$. A similar objective is used for decoupling of the light from the fiber. Application of such an objective is required because of the high numerical aperture of the fiber resulting from high refractive index contrast between the core and the cladding. With our experimental setup we achieve $\approx 47 \%$ transmission through the fiber. Measurements of the spatial distribution of the optical fields emerging in the fiber are performed with a beam profiler (BC106 from ThorLabs) and an additional optical system (not shown in Fig. 1).
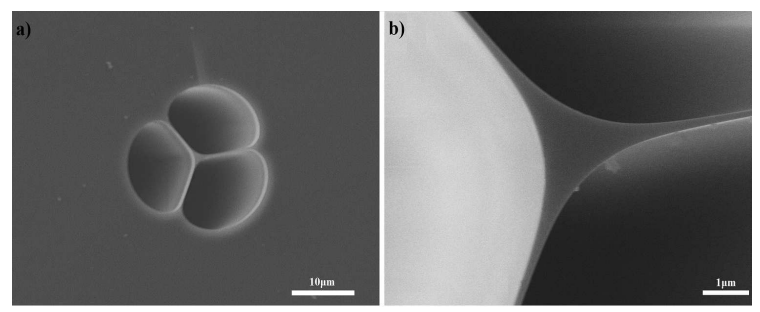

Fig. 2. (a) Cross-section of the examined suspendedcore fiber, (b) core of the fiber.

A model of the fiber used for numerical simulation is shown in Fig. 3. Simulations are performed with geometric parameters of the core, struts, and holes extracted from experimentally measured values with a refractive index of 1.453 characterizing silica at $795 \mathrm{~nm}$. The sim- ulations are performed with a perfectly symmetric structure of the fiber that posses 3 -fold rotational symmetry. It should be noted, however, that the real fiber is not perfectly symmetric (difference in sizes of the struts), which may be one of the sources of small discrepancy between experimental data and simulations.

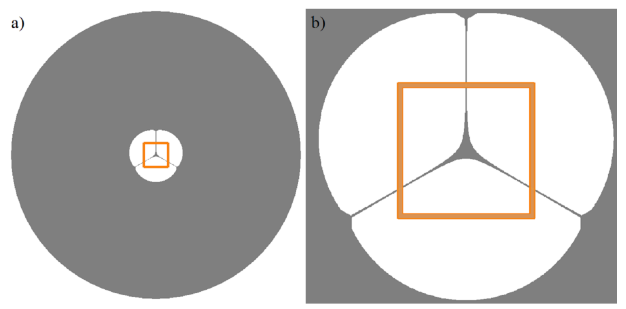

Fig. 3. Model fiber used in simulations: (a) cross-section of the whole fiber, (b) the core area. Squares on the pictures mark the $10 \times 10 \mu \mathrm{m}$ simulation area. Core diameter: $125 \mu \mathrm{m}$, struts' width: $135 \mathrm{~nm}$.

To simulate propagation of light in the SCF, a fully-vectorial mode solver (Lumerical Mode Solutions) is used. A whole region of simulation, shown as an orange rectangle in Fig. 3, is $10 \mu \mathrm{m}$ square with a $600 \times 600$ mesh, which yields a distance between simulation nodes equal to $16.6 \mathrm{~nm}$. On the borders of the simulation region perfectly matched layers boundary conditions are applied.

\section{Results}

In our analysis we concentrate on three properties of the fiber: mode structure, numerical aperture, and coupling efficiency. Their detailed discussion is presented below.

\subsection{Mode structure}

Numerical simulations reveal 24 different transverse modes. Six of them are presented in Fig. 4. The modes form a doublet-singlet series, i.e. two first modes, shown in Fig. 4a and b, constitute a doublet (the same intensity distribution but different polarization), then there is a non-degenerate single mode (Fig. 4c), which precedes another doublet (Fig. 4d and e). In a perfectly symmetric structure, no birefringence between doublet modes should be observed [13] hence they should be characterized with the same effective refractive indices. In our simulations, however, a residual birefringence is observed. It arises from special discretization of the model and refractive index, i.e. with a rectangular mesh, distribution of the refractive index along one vertical strut (which follow meshing direction) will be slightly different than for other two struts. This difference lifts the degeneracy of the two modes leading to a "numerical" birefringence on order of $10^{-6}$ which sets the limit for simulation accuracy.

In order to quantitatively analyze the fiber, we compare numerical results with real profiles measured at the 


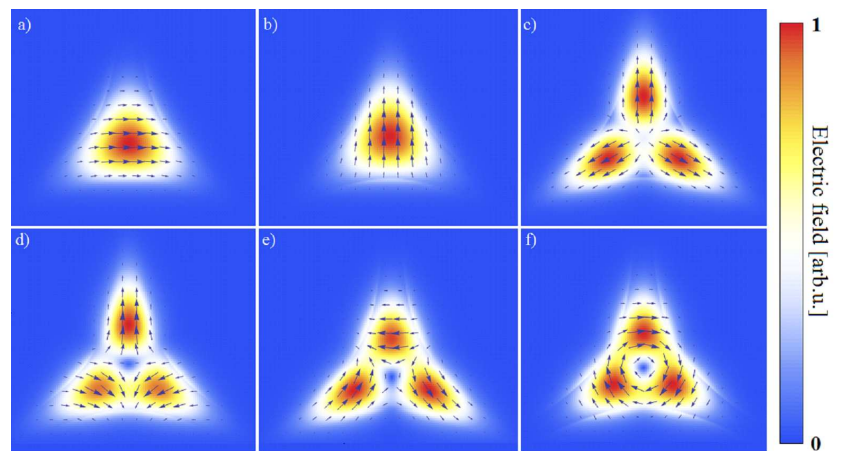

Fig. 4. Electric field distributions of the simulated modes: (a), (b) first double (effective index $n_{\text {eff }}=$ $1.4078)$, (c) first singlet $\left(n_{\mathrm{eff}}=1.3614\right)$, (d), (e) second doublet $\left(n_{\mathrm{eff}}=1.3543\right)$, (f) second singlet $\left(n_{\mathrm{eff}}=\right.$ 1.3515). The arrows represent the $\boldsymbol{E}$ vectors and the light intensities are represented by a color scale.
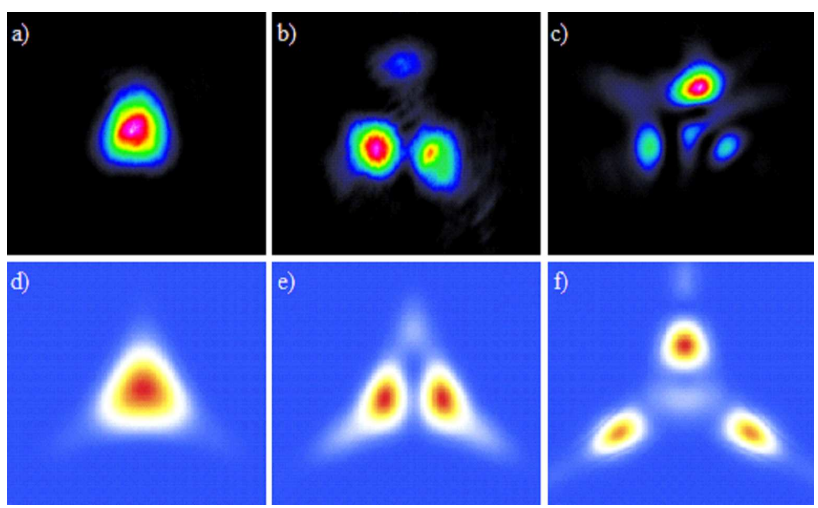

Fig. 5. Measured (a)-(c) and modelled (d)-(f) optical power density profiles of the outgoing beam: (a), (d) linearly polarized light focused at the center of the fiber's core, no dependence on light polarization, (b), (e) and (c), (f) non-central coupling to the fiber, higher modes are excited, resulting in the field distribution highly dependent on light polarization.

fiber's end. Exemplary distributions are shown in Fig. 5. These profiles are superpositions of different modes with appropriate amplitudes and respective phases. For instance, the first profile (Fig. 5a) is obtained with a light beam illuminating the center of the fiber front normal to the fiber-face surface. This case allows maximum coupling of the light into the fiber (the highest power measured at the other end of the fiber) and can be easily associated with the first order mode; if there is no displacement between the fiber core center and the focal spot of the beam, the fundamental mode is most efficiently generated. This observation is confirmed numerically (Fig. 5d)). In that case, efficiency of light coupling into the fundamental mode reaches as much as $92 \%$ of the total transmitted power. It is noteworthy that the light field distribution in the mode is independent of the light polarization. For non-central and/or non-normal il- lumination of the fiber front face the fundamental mode is not most efficiently generated (Fig. 5b). Moreover, in that case the coupling efficiency also depends on the polarization of the incident beam. In order to verify that such a distribution is consistent with our simulation we sum over a few special modes that are excited with a light beam focused on a vertical strut $0.5 \mu \mathrm{m}$ below the fiber core center. The field was simulated for a fiber of a length of $60 \mathrm{~cm}$, which corresponds to the length of a measured fiber. As shown (Fig. 5), simulations reproduce the measurements. In particular, they reveal similar dependence of the mode on the polarization of the incident light.

\subsection{Numerical aperture}

Numerical aperture (NA) is the second parameter we were concerned with. In order to perform the measurements, we mounted the fiber end at a goniometer table and placed a detector right behind the fiber (decoupling objective shown in Fig. 1 as $\mathrm{O} 2$ was removed). Based on an angular dependence of the light power and a Gaussian fit to the data (Fig. 6), we obtained NA $=0.8640(79)$ at the $5 \%$ level and $\mathrm{NA}=0.7890(55)$ at the $10 \%$ level.

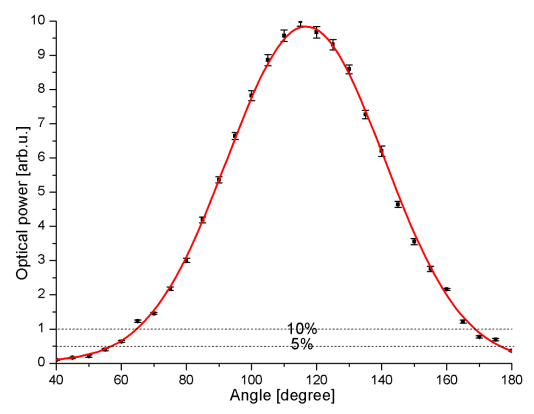

Fig. 6. Angular dependence of the measured light power. Red curve is a Gaussian fit to the measured data.

\subsection{Coupling to the fiber}

In our experiment we use a fiber with a core size comparable to the wavelength of light. In that case, even a small displacement of the focus on the fiber end changes the coupling conditions, which, in particular, leads to the generation of higher order modes. To describe the problem theoretically, we compute the power coupling coefficients corresponding to fractional powers present in given modes. For that case we decompose the light electric field $E$ and magnetic field $H$ in orthogonal base of $e_{i}$ and $h_{i}[14]$ :

$$
E=\sum_{i} a_{i} e_{i}, \quad H=\sum_{i} b_{i} h_{i}
$$

where

$$
a_{i}=\frac{\int \mathrm{d} S E \times h_{i}^{*}}{\int \mathrm{d} S e_{i} \times h_{i}^{*}}, \quad b_{i}=\frac{\int \mathrm{d} S e_{i} \times H^{*}}{\int \mathrm{d} S e_{i} \times h_{i}^{*}},
$$

and 


$$
\frac{\int \mathrm{d} S e_{i} \times h_{j}^{*}}{\int \mathrm{d} S e_{i} \times h_{i}^{*}}=\delta_{i j} .
$$

Assuming that no power is reflected from the front of the fiber, the total power transmitted through it is equal to

$$
\begin{aligned}
P & =\frac{1}{2} \Re\left(\int \mathrm{d} S E \times H^{*}\right)=\frac{1}{2} \Re\left(\int \mathrm{d} S E_{0} \times H_{0}^{*}\right) \\
& =\frac{1}{2} \sum_{i} \Re\left(a_{i} b_{i}^{*} \int \mathrm{d} S e_{i} \times h_{i}^{*}\right),
\end{aligned}
$$

where $E_{0}$ and $H_{0}$ describe the electric and magnetic field of incident light. Each element of the sum represents power in the $i$-th mode. Thus a power coupling coefficient for different modes is given by

$$
\begin{aligned}
\frac{P_{i}}{P} & =\Re\left(\frac{\int \mathrm{d} S e_{i} \times H_{0}^{*} \int \mathrm{d} S E_{0} \times h_{i}^{*}}{\int \mathrm{d} S e_{i} \times h_{i}^{*}}\right) \\
& \times \frac{1}{\Re\left(\int \mathrm{d} S E_{0} \times H_{0}^{*}\right)} .
\end{aligned}
$$

It should be noted that in reality a fraction of light is reflected from a fiber's front face. For each mode, the reflected fractions are slightly different due to a refractive index mismatch (for more details see Ref. [13]). Since in our case less than $3 \%$ of the fundamental mode is reflected, it is negligible. For the simulations we assume a Gaussian profile of the light beam with half width at half maximum equal to $1 \mu \mathrm{m}$, which is a calculated beam waist for a beam obtained from $60 \times$ objective. Moreover, we assume normal incidence of light onto the fiber's facet.

The calculations are performed for two different situations: (a) when one focuses the light directly in the center of the fiber's core and (b) when there is non-zero displacement between them.

\subsubsection{Perfect overlap between fiber core and light beam}

We first consider maximum coupling of the light into the fiber (Fig. 5a). Conditions for such coupling are

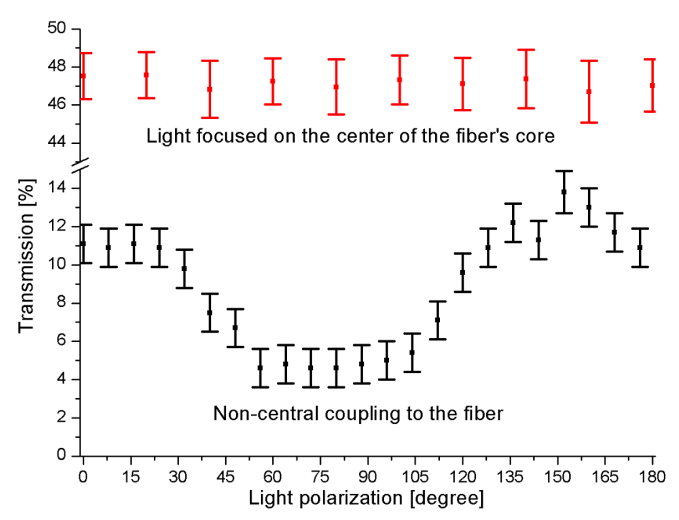

Fig. 7. Measurements of the transmission dependence on the incoming light polarization. For perfect coupling conditions no dependence is visible within experimental accuracy.

achieved for central and normal illumination of the fiber core's center. It is demonstrated experimentally that in the case up to $47.65(17) \%$ of the total power is transmit- ted through the fiber. Most of the power is present in the two degenerate fundamental modes. The simulations show that as much as $92 \%$ of the total power is coupled to the modes. Amplitudes of all other modes are negligible. It should be noted that in the case of most efficient coupling the total power transmitted through the fiber does not depend on incoming light polarization (Fig. 7).

3.3.2. Non-zero displacement between fiber's core and light beam

Focusing light not exactly at the center of the fiber's core introduces polarization dependence of both, the total and modal coupling coefficients. While small displacements cause differences only at the level of the mode distribution, larger shifts may lead to dependence of the whole coupling efficiency on light polarization. For example, when light is focused $0.5 \mu \mathrm{m}$ above the core center (closer to the vertical strut), the optical field distribution becomes polarization sensitive (see Table I). At the same time the total transmitted power is independent of that light polarization.

In case of stronger displacements, e.g., $1.5 \mu \mathrm{m}$, the situation changes (Table II) and the total coupling efficiency, and thus the total transmitted power, becomes light-polarization dependent (Fig. 7). Such displacements also corresponds to much smaller total transmitted power $(\approx 8 \%$ in average) than it was observed in previous cases. It results from imperfect coupling to the fiber, in particular, more efficient generation of strongly attenuated higher-order modes.

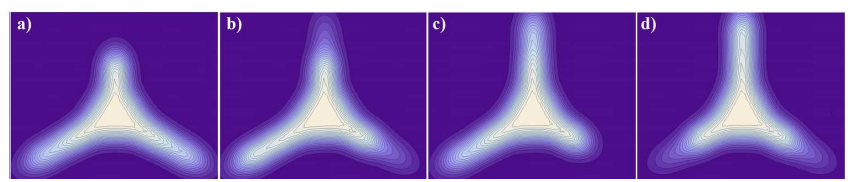

Fig. 8. Simulated coupling maps calculated for different incoming light polarizations: (a) horizontal $-0^{\circ}$, (b) rotated by $30^{\circ}$, (c) rotated by $60^{\circ}$, (d) vertical $90^{\circ}$.

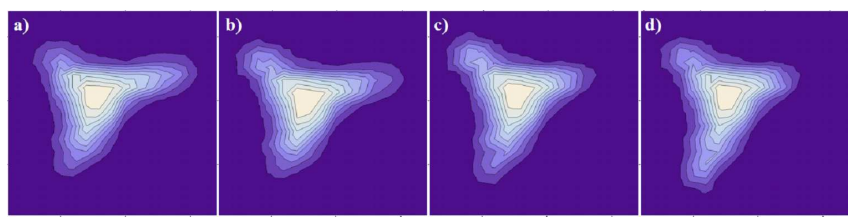

Fig. 9. Coupling maps measured for different incoming light polarizations (with azimuth changing from $0^{\circ}$ to $90^{\circ}$ with $30^{\circ}$ step). Since fiber orientation was not known, polarizations are not exactly specified.

Coupling efficiencies can also be presented in the form of coupling maps, where the total coupling efficiency is plotted against the displacement on both $x$ and $y$ axis. This quantity is especially interesting because it may be both simulated and directly measured. 
TABLE I

Coupling coefficients for the case of the focusing light $0.5 \mu \mathrm{m}$ above the core center. Coefficients are given for the first twelve modes (RM - remaining modes), coupling to higher order modes is negligible (approximately $2 \%$ ). Polarization 90 refers to the light polarized along the vertical strut.

\begin{tabular}{|c|c|c|c|c|c|c|c|c|c|c|c|c|c|c|}
\hline \multirow{2}{*}{$\begin{array}{c}\text { Polarization } \\
\text { [degree] }\end{array}$} & \multirow{2}{*}{$\begin{array}{l}\text { Total coupling } \\
\text { efficiency [\%] }\end{array}$} & \multicolumn{13}{|c|}{ Mode coupling efficiency [\%] } \\
\hline & & 1 & 2 & 3 & 4 & 5 & 6 & 7 & 8 & 9 & 10 & 11 & 12 & $\mathrm{RM}$ \\
\hline 0 & 96 & 37 & 0 & 0 & 0 & 27 & 24 & 0 & 6 & 0 & 0 & 0 & 0 & 2 \\
\hline 30 & 96 & 28 & 12 & 6 & 5 & 20 & 18 & 0 & 5 & 0 & 0 & 0 & 0 & 2 \\
\hline 60 & 96 & 9 & 37 & 18 & 15 & 7 & 6 & 0 & 2 & 1 & 1 & 0 & 0 & 0 \\
\hline 90 & 96 & 0 & 50 & 24 & 20 & 0 & 0 & 0 & 0 & 1 & 1 & 0 & 0 & 0 \\
\hline
\end{tabular}

TABLE II

Coupling coefficients for the case of the focusing light $1.5 \mu \mathrm{m}$ above the core center. Coefficients are given for the first twelve modes (RM - remaining modes), coupling to higher order modes is negligible (approximately $2 \%$ ). Polarization 90 refers to the light polarized along the vertical strut.

\begin{tabular}{|c|c|c|c|c|c|c|c|c|c|c|c|c|c|c|}
\hline \multirow{2}{*}{$\begin{array}{c}\text { Polarization } \\
\text { [degree] }\end{array}$} & \multirow{2}{*}{$\begin{array}{c}\text { Total coupling } \\
\text { efficiency [\%] }\end{array}$} & \multicolumn{13}{|c|}{ Mode coupling efficiency [\%] } \\
\hline & & 1 & 2 & 3 & 4 & 5 & 6 & 7 & 8 & 9 & 10 & 11 & 12 & $\mathrm{RM}$ \\
\hline 0 & 67 & 0 & 0 & 0 & 0 & 1 & 1 & 0 & 3 & 0 & 0 & 10 & 8 & 44 \\
\hline 30 & 72 & 0 & 0 & 1 & 1 & 1 & 1 & 5 & 2 & 4 & 2 & 7 & 6 & 42 \\
\hline 60 & 81 & 0 & 0 & 2 & 4 & 0 & 0 & 14 & 1 & 12 & 7 & 3 & 2 & 36 \\
\hline 90 & 85 & 0 & 0 & 3 & 5 & 0 & 0 & 19 & 0 & 17 & 10 & 0 & 0 & 31 \\
\hline
\end{tabular}

The spatial resolution of the simulations (Fig. 8) is equal to $11.6 \mathrm{~nm}$ and a whole simulation area of $6.1 \times$ $6.1 \mu \mathrm{m}^{2}$ is divided into a $53 \times 53$ mesh. In case of measurements, spatial step equals to $500 \mathrm{~nm}$ and 17 points are taken, which yields a total area of $8 \times 8 \mu \mathrm{m}$ (Fig. 9). Both simulated and experimental results exhibit a polarization dependence. As it was earlier stated, it is observed only when the light beam is focused exactly at the fiber's center. During the measurements the orientation of the fiber was not known, so it is hard to directly compare the experimental data and results of the simulations. However, it may be shown from geometrical arguments that rotating light polarization by $60^{\circ}$ leads to a tilt of the observed profile (pairs (a)-(c) and (b)-(d) in Fig. 8 and (a)-(c) in Fig. 9).

Most asymmetric profiles (Figs. 8d and 9d) exhibit many similarities, hence both may refer to the same coupling conditions. Several parameters of those distributions can be estimated in order to compare them. Both profiles stay in very good agreement with the measurements (Table III). The most important conclusion is that displacement as small as $\approx 1.5 \mu \mathrm{m}$ will cause significant reduction of the coupling efficiency. We attribute the resulting differences to the incoming beam shape. We assumed its width based only on the objective's numerical aperture. In addition, it is very difficult to set an incoming beam perfectly perpendicular to the fiber's end and focus exactly on it.
TABLE III

Comparison of different parameters of the measured and calculated distributions.

\begin{tabular}{c|c|c|c|c}
\hline \hline & $a[\mu \mathrm{m}]$ & $b[\mu \mathrm{m}]$ & $c[\mu \mathrm{m}]$ \\
\hline simulated & 4.00 & 3.20 & 1.20 \\
measured & $4.08(41)$ & $3.37(41)$ & $1.84(41)$ &
\end{tabular}

\section{Conclusions}

In this work we have investigated the suspended-core microstructured optical fiber. The fiber exhibits a very high numerical aperture which originates from high refractive-index contrast built-in into the fiber structure. The structure is also responsible for a high number of modes that may be excited. As shown, coupling coefficients of respective modes strongly depend on the light coupling conditions (placement of the focus on the fiber's structure and, in some cases, light polarization). In order to optimize transmission through the fiber, one has to couple light into the fundamental mode. It may be achieved by precise focusing of the light onto the center of the fiber's core. If this condition is not met, power coupling to the fundamental mode becomes less efficient, and higher order modes are excited. Moreover, it was demonstrated experimentally that for displacements smaller than $1 \mu \mathrm{m}$, there is no measurable dependence of the coupling on the light polarization. For larger displacements, however, such dependence was observed. It was also shown that in the later case the total power of transmitted light depends on light polarization. 
Our results are important in context of design and fabrication of suspended-core fibers dedicated for spectroscopic measurements and/or gas or liquid sensing. We demonstrated that coupling conditions, e.g. spatial overlap of light beam and fiber's core and light polarization, determines efficiency of coupling light into particular fiber's modes. Since each mode has different fractional power present in the holes, it offers a control over the strength of interaction between light and medium filling the holes. In particular, this interaction may be enhanced. The problem of interaction of light and media filling the holes will be the object of our further research.

\section{Acknowledgments}

The authors would like to express their gratitude to Prof. Wacław Urbańczyk and Dr. Tomasz Nasiłowski for stimulating discussions and encouragement to start a research program on photonic-crystal fibers. The authors also express acknowledgments to Dr. Elżbieta Bielańska for her help in taking SEM images of the fiber. This work was partially supported by the European Union within the Innovative Economy Framework Programme "Team Project" operated by the Foundation for Polish Science and Polish Ministry of Science and Higher Education (grant N N202 074135).

\section{References}

[1] P. Russell, Science 299, 358 (2003).

[2] A. Fernando, E. Silvestre, P. Andres, J.J. Miret, M.V. Anders, Opt. Express 9, 687 (2001).

[3] W.J. Wadsworth, R.M. Percival, G. Bouwmans, J.C. Knight, P.St.J. Russell, Opt. Express 11, 48 (2003).
[4] D.G. Ouzounov, F.R. Ahmad, D. Muller, N. Venkataraman, M.T. Gallagher, M.G. Thomas, J. Silcox, K.W. Koch, A.L. Gaeta, Science 301, 1702 (2003).

[5] M.N. Petrovich, A. van Brakel, F. Poletti, K. Mukasa, E. Austin, V. Finazzi, P. Petropoulos, E. O'Driscoll, M. Watson, T. DelMonte, T.M. Monro, J.P. Dakin, D.J. Richardson, Proc. of SPIE 6005, 60050E (2005).

[6] Y.L. Hoo, W. Jin, H.L. Ho, D.N. Wang, R.S. Windeler, Opt. Eng. 41, 8 (2002).

[7] T.M. Monro, D.J. Richardson, P.J. Bennett, Electron. Lett. 35, 1188 (1999).

[8] T. Pustelny, M. Grabka, Acta Phys. Pol. A 114, A113 (2008).

[9] T. Pustelny, M. Grabka, Acta Phys. Pol. A 116, 385 (2009).

[10] T.G. Euser, J.S.Y. Chen, M. Scharrer, P.S.J. Russell, N.J. Farrer, P.J. Sadler, J. Appl. Phys. 103, 103108 (2008).

[11] Y. Ruan, T.C. Foo, S. Warren-Smith, P. Hoffmann, R.C. Moore, H. Ebendorff-Heidepriem, T.M. Monro, Opt. Express 15, 18514 (2008).

[12] J. Wojcik, P. Mergo, M. Makara, K. Poturaj, L. Czyzewska, J. Klimek, A. Walewski, in: Photonic Crystal Fibers II, Eds. K. Kalli, W. Urbanczyk, Spie-Int. Soc. Opt. Eng., Bellingham 2008, Vol. 6990, p. T9900.

[13] M.J. Steel, T.P. White, C.M. de Sterke, R.C. McPhedran, L.C. Botten, Opt. Lett. 26, 488 (2001).

[14] A.W. Snyder, J. Love, Optical Waveguide Theory, Chapman and Hall, London 1983. 